\section{Original Commmaxations.}

\section{ON THE USE OF THE SPIIYGMOGRAPII IN THE INVESTIGATION OF DISEASE.}

By Batmeazar W. Foster, M.D., M.R.C.P.Tond. Licentiate of King and Queen's College of Physicians; Physician to the Queen's Hospital, and Professor of Clinical Medicine in Queen's College, Birmingham; etc.

[Dead before the Midland Medical Society, Fetruary 6th,1806.]

\section{Part II.}

Or late years, our knowledge of the pathology of the organs of the circulation has been much extended, and our power of detecting their morbid conditions has reached a point of much excellence; yet all will admit that there are many diseases of which we are even now but slightly informed, and many cases in which our power of diagnosing the exact cardiac lesion is often unsatisfactory. Many of these cases are examples of the early stages of maladies of which we know but too well the more developed phases. Many others are the slighter forms of organic change in which the funotions are but little disturbed. By means of the sphygmograph of Marey we can detect changes in the movement of the blood that would ordinarily escape us; and by its use, I have no doubt we shall, when our observations have multiplied suffciently, gain much information concerning morbid conditions in their earlier stages, as well as a knowledge of the slight indications capable of assisting in prognosis.

In the better understood diseases of the heart and great vessels, we can arrive at a diagnosis by the aid of the ordinary means employed; but in most cases the instrument of Marey can afford us useful confirmatory evidence, and in many it can perfect a diagnosis which percussion and auscultation have failed to complete. The pulse-trace alone often enables those skilled in its interpretation to foretel the sounds to be heard on auscultation; and in doubtful cases, by the information it yields, augments in no small degree the certainty of the diagnosis.

In the remainder of the time allotted to me, it will be my endearour to point out a few of the morbid states of the organs of circulation, in which the pulseform affords valuable indications. In doing so, I shall chiefly confine myself to diseased conditions of the organs of circulation, because I have had in such conditions the most extensive opportunities for investigation.

Among the pathological changes with which the arteries may be affected, is one that we so often meet with in old age, that we almost deem it the condition natural to our last days. I allude to that Senile Change in the Vessels which gives to the finger placed over the radial artery the sensation of resting on a hard inelastic tube. With such change in the arteries, we usually find hypertrophy of the left ventricle, and also a dilated state of the vessels themselves. We can easily account for these associated conditions, when we reflect that by such alteration the elasticity of the arterial walls is more or less destroyed. The special property, indeed, by which the arteries facilitate the onward flow of the blood and regulate its movement, is gradually lost; and th the heart, thus deprived of one of its chief aids, is compelled to act more forcibly in order to carry on. the circulation. Hypertrophy of the left ventricle is $C$ the consequence; and, little by little, the degener- $\overrightarrow{\overrightarrow{7}}$ ated ressels, having lost their facility of modifying $\frac{\text { ? }}{+}$ the force of the heart, dilate under the increased energy with which the blood is impelled into them.

Thanks to the luminous descriptions of Virchow, $\overline{\bar{\omega}}$ we are now well acquainted with forms of degenera- $\frac{5}{T}$ tive change other than ossification affecting the great $\propto$ arterial trunks; and we know also that such changes, o as well as ossification, may occur at a comparatively on early period of life. All these changes may extend $\vec{O}$ over more or less of the vascular system; but they all have one common effect-they diminish the elas- $\vec{\omega}$ ticity of the arterial wall.

This deterioration of a necessary property entails $\delta$ not only the changes to which I have above referred, 3 . but it is associated with an ever threatening tend.ency in the degenerated vessels to rupture under any iv abnormal pressure of the blood.

The power of detecting such alteration when it affects the larger trunks, leaving the superficial ves- W sels healthy, would be a great boon to practical medicine, by enabling us to recognise with certainty a condition which we can now only vaguely surmise. $\omega$ The sphygmograph will assist us in this; for we find that certain forms of the pulse-trace are character. 3 istic of senile degeneration of the vessels; and the appearance of these peculiarities in a minor degree $\stackrel{\rho}{工}$ in early life leads us to conclude that arterial chango $\overrightarrow{ }$ has occurred. The elasticity of the arteries trans- $\infty$ forms, as we know, the movement given to the blood $\sigma$ by the ventricular systole; and, therefore, we find. the least trace of the ventricular movement in the arteries most distant from the heart. But, whenever the arterial elasticity is diminished, the pulse retains more or less of the form given by the ventricular contraction.

M. Marey, by an ingenious experiment (op. cit., $\stackrel{\mathbb{\perp}}{\varrho}$ p. 416), has obtained the trace of the ventricular $\vec{F}$ aystole (in a horse), which is figured below. In the diagram (Fig. 1), the dotted continuation indicates

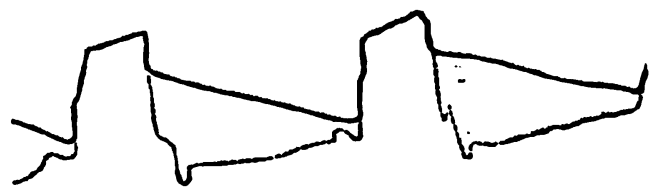

Fig. 1.

the traces of the ventricle, while the unbroken line shows the form which the arterial pulsation should possess in senile degeneration of the ressels. In the. following traces, the resemblance to the ideal form $N$ is sufficiently striking. (Figs. $2,3,4$.)

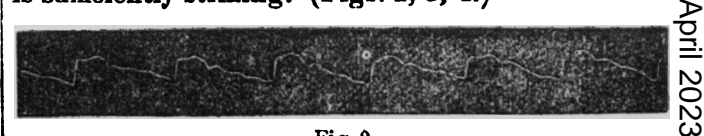

Fig. 2.

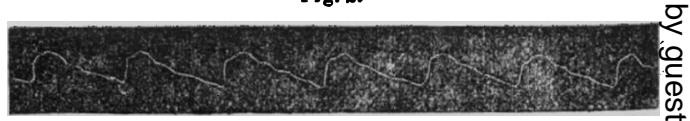

Fig. 3.

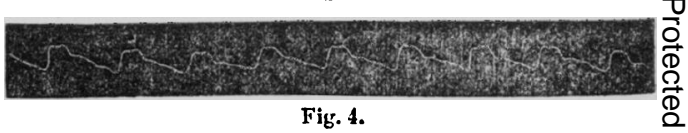

On examination, we recognise the following as the chief characters of the senile pulse : the great amplitude of the trace; the vertical and sometimes brokeno 
line of ascent; the extended summit; the sudden fall of the first part of the line of descent ; and its freedom from dicrotism.* These peculiarities are easily accounted for by the loss of elasticity in the vessels, the increased energy of the ventricle, and the dilatation of the arteries themselves. These features, when found in a minor degree in the pulse-trace of one little advanced in life, justify the opinion that there is arterial change. The rounded summit and the diminution of dicrotism are, when well marked, the first signs of alteration of the arterial elasticity. M. Marey has been able to test the diagnostic value of these characters by a post mortem examination. The pulse-trace (Fig. 5), taken by me in a case in

Fig. 5.

which considerable atheromatous change of the aorta and many of the larger vessels was found after death, shows the features of the trace of arterial degeneration in its more limited form. The trace (Fig. 6)

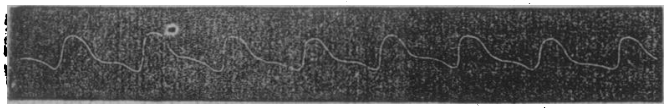

Fig. 6.

well shows the form of pulsation which excites suspicion of commencing arterial change. It was taken from a patient (age 37 ) in whom many symptoms lead to the diagnosis of this condition. The pulse-trace of strong tension simulates slightly that of senile change ; but it is easy to discover if any of the causes of increased tension are acting.

In the investigation of Aneurisms, we shall find that the sphygmograph can afford us much valuable service, by showing us in the pulse-trace the modifications in the movement of the blood produced by the diseased condition.t These modifications are intimately dependent on the seat of the tumour, its size relatively to the vessel with which it is connected, and the elasticity of its walls.

We may, following the arrangement of the French author, consider, first, aneurism so situated on an artery that the pulse can be observed on the vessel below the tumour; and secondly, aneurisms affecting the aorta. The beat of an artery, when carefully felt below an aneurismal tumour which implicates it, is found to present unusual characters ; it is weakened, and generally retarded. These changes are due chiefly to the fact that the blood has to traverse a sac whose elasticity much modifies the movement of the fluid, as Marey has proved by artificially producing the conditions in a series of experiments. By means of the sphygmograph, we obtain traces which poivt out that modifications of the pulse occur both in its form and force. In the pulse collected on an artery below an aneurism, we observe the changes which are the result of the transforming influence of the elasticity of the sac: the movement of the blood in the vessel approaches rather to that which is normally seen in the smaller arterial branches. The vertical line of ascent disappears; and often this line approaches in length that of descent. Thus we have a more feeble pulsation given to the finger; and, as the summit is slow to occur, there is further

- I am indebted to the kindness of Dr. F. Robinson, Medioal Officer of the Birmingham Workhouse, for the opportunity of obtaining the traces (Figs. 2, 3, 4) from inmates between 80 and 90 years of age.

(in Lancet, Jan. 20th, 1866) a case in which valuable in formation was gained from a consideration of pulse-traces by Drs. Sanderson and Anstie. a sensation of apparent retardation felt in addition. In the following figures $(7,8)$ we see an example, in (n) Fig. 7.-Left radial.

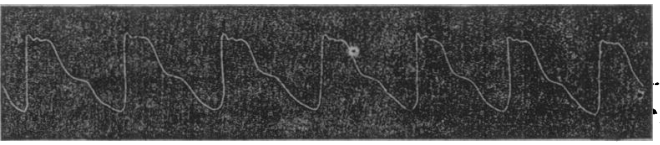

Fig. 8.-Kight radial.

the pulse-trace of the left radial artery, of the modifying influence of an elastic aneurismal sac. The traces were taken from a patient in the Queen's Hospital (under the care of my colleague Dr. Fleming), affected with an aneurism of the left subclavian artery, within the thorax. The right pulse shows very well the trace of another lesion under which the man labours; viz., insufficiency of the aortic valves. So characteristic of the conditions in this case were the above traces, that they alone enabled me to arrive at the diagnosis, to which ordinary means had led those watching the case. At the time when the above traces were taken, the tumour was evidently large and very elastic : hence the great modifications in the pulse-form produced. I have, by the kindness of Dr. Fleming, had opportunities of examining this patient from time to time; and, since hehas been under treatment, and has been kept strictly at rest, the traces taken below the aneurism now show much less modification in the pulse-form than formerly. (Fig. 9.) This we may attribute to

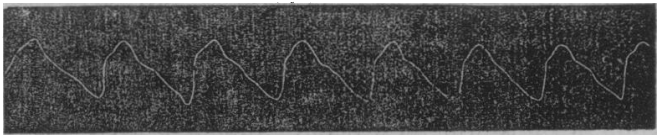

Fig. 9.-Left radial.

the diminution of the elasticity of the sac, following either deposition of fibrine on its interior, or the contraction of adhesions with neighbouring parts. In this case, the former supposition is rather warranted by the changes perceptible in the tumour.

In Aneurisms of the Aorta, we find that very much less striking indications are afforded by the pulsetrace. Those pointed out by Marey are: 1, modifications in the force of the pulse; 2 , modifications in the intensity of the dicrotism; and 3 , the existence of a constant difference in the pulse-form. of the two radial arteries.

1. The force of the pulse, according to the French writer, is seldom much diminished. This character is only of small value in diagnosis. The causes of this want of change in force reside probably $(a)$ in the small size of the tumour relatively to the volume of the aorta; (b) in the fact that the sac is sometimes not placed in the direct route of the blood, but communicates with the vessels, and thus has a much less transforming effect upon the blood-movement, than a tumour which must be traversed by the current; and $(c)$ in the thickness and slight elasticity of the wall of the sac, often found in aneurisms in this situation. The force of the pulse, too, in these cases is altered very often, not in one artery alone, but in the vessels of both sides of the body; and, on the other hand, it must be remembered that the tumour, by compressing the orifice of one of the branches of the aorta, may cause a peculiarity in the radial of one side.

2. The modification in the dicrotism may exist in one or both radial pulses, and is occasionally a sign of vuuch value. 
3. The presence of a constant dissimilarity in the pulse-traces of the radial arteries is the most valuable aign in the diagnosis of aortic aneurisms. In many cases, the finger can perceive a want of parallelism in the beats of the radials, but often this is too slight to be detected by the finger. It sometimes shews itself in the trace by a slight difference in the dicrotism only; at others, the difference in form is more evident. When the tumour is so situated that it can be handled, we can gain valuable evidence as to its nature by observing the changes in the tension of the arteries, produced by its alternate compression and relaxation. The traces (Figs. 10, 11, 12, 13)

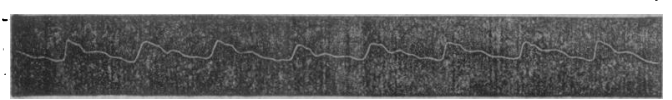

Fig. 10.-Left radial.

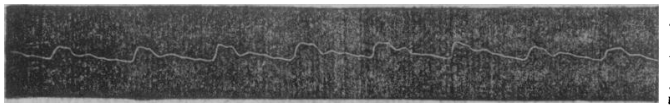

Fig. 11.-Right radial.

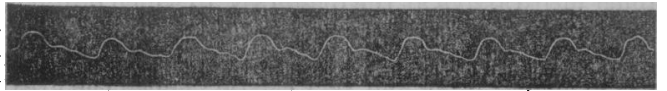

Fig. 12.-Right radial.

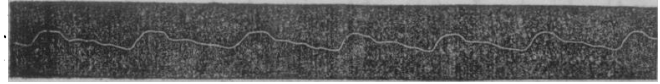

Fig. 13.-Left radial.

have been taken from patients suffering under intrathoracic aneurisms; and the want of any great modification in the pulse-form of either radial has led me to consider the lesions as aortic.*

The detection of Organic Diseases of the Heart is rendered so certain by the use of auscultation and percussion, that we have but in few cases need of any other aid. In some cases, however, the pulseevidence is useful; and this can be much more accurately and usefully obtained by the use of the sphyg. mograph than by any other means. I can now only briefly allude to certain affections of the cardiac orifices; and in these cases we shall find useful information yielded us by the instrument.

The changes of the aortic orifice to which I shall allade are those involving obstruction to the free exit of the blood from the ventricle, and those which allow of regurgitation. The first lesion is frequently met with, especially in the old, and produces often few symptoms, especially when it has gradually supervened, and the ventricle has had time to hypertrophy and counteract its effects. The pulse is generally regular; and the peculiarity observed in the trace is the obliquity of the line of ascent, which marks the greater duration of the ventricular systole and the gradual entry of the blood into the vessels. In the traces below (Figs. 14, 15), these characters are seen ; but, as the second was taken from an old woman of

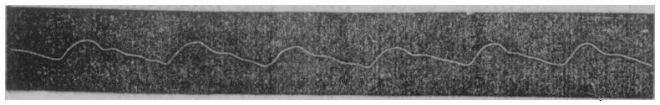

Fig. 14.

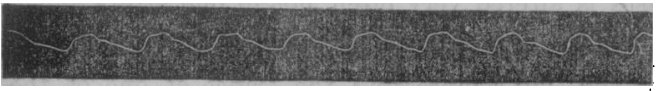

Fig. 15.

* I am indebted to Dr. Russell for an opportunity of seeing the patfent from whom Figs. 10 and 11 were taken; and I have also to xpress my thanks to Drs. Gasey and Steell for their kindness in assisting $\mathrm{me}$ in examining patients at the General Hospital. over eighty, the modifications of senile change superadded.

Aortic insufficiency, or incompetency of the sigmoid valves, produces certain effects of a very striking character in the pulse-beat. These have been well: described by Corrigan (to whom, indeed, our knotw. ledge of the disease is chiefly due), and consist mainf in the jerking force of the pulse, its visible chis. racter, and the peculiar sensation given by it to the finger. These peculiarities are consequences of theregurgitation of the blood into the ventricle durifig its diastole. Marey has from his experiments cofcluded that the force of the pulse is not due altogether to the ventricular hypertrophy which accompanies the lesion, but that the amplitude of the trace and apparent force of the pulse are due really to the feeble tension of the arterial wall, which exists in consequence of the regurgitation during the ventcular diastole. In the first part of this paper, traees illustrating the effect of this feeble tension in increasing the amplitude of the pulsations have beta figured. The traces (Figs. 16, 17), collected from pto-
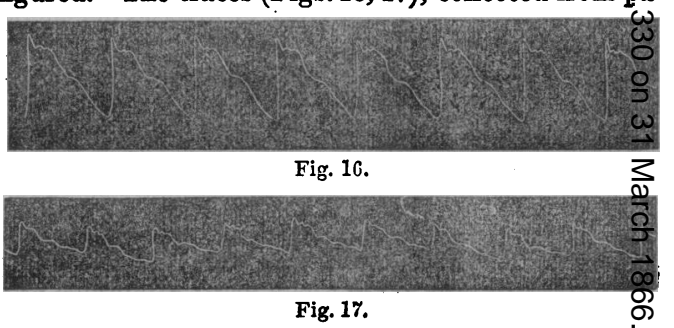

tients suffering from aortic patency, are marked by the following peculiarities. There is great amplitudeof trace, as just explained. The vertical line of ascent marks the sudden ventricular contraction; this vertigal line is terminated by a sharp-pointed process, whioh indicates that, on account of its very sudden elefation, the lever has, by virtue of an acquired quickness, ceased to be connected for a brief interval with the arterial movements. The summit of the pulsh. tion is in many cases very short; but in others it presents a horizontal or curved line, especially if any constriction of the aortic orifice, or other cause prieducing delay of the passage of the blood into the ve sels, obtain.

The trace (Fig. 17) was taken in a case of aor to patency, complicated with senile change in the vessels.

In mitral insufficiency, the irregularity of to pulse so well known may be registered very accirately by the sphygmograph. This irregularity is. its chief characteristic, and, when recorded, is vewy interesting.

In the traces below (Figs. 18, 19, 20), collected froin

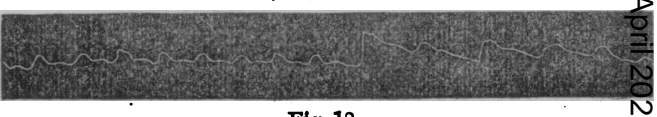

Fig. 18.

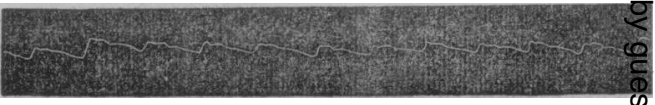

Fig. 19.

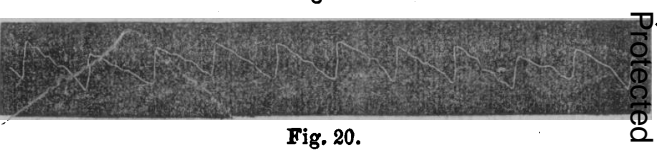

Fig. 20.

cases of this disease, that numbered 20 was compt. cated with the signs of contraction of the auricula. 
ventricular orifice. This additional lesion nearly always diminishes the irregularity of the pulsecharacteristic of pure mitral regurgitation.

In conclusion, I would call attention to the trace (Fig. 21) taken from a man suffering under lead-

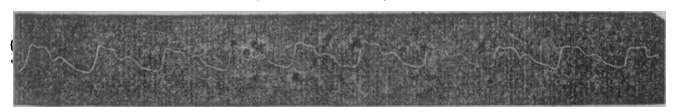

Fig. 21.

poisoning; it presents a peculiarity of form which has been figured by Marey, and which, when I saw it in his book, I scarcely expected to be able to verify so strikingly. I have since collected traces in several cases of this nature, and in all have met with this peculiar form. It may be indicated now as a curious fact : the explanation of it further investigation may discover. The subject of the pulse-form in acute disease, I may also add, is full of interest, and will, I am confident, from the little I have already seen of it, yield much interesting and useful information. This subject is receiving the attention of Dr. Anstie; and from his investigations, which were alluded to in the Lancet some months back, we have reason to expect some very valuable information. In now closing these remarks, I trust that this exposition of the views of the French physiologist may have not only proved interesting, but may have also indicated a means by which we can render our knowledge of disease more accurate, and at the same time pointed out a field of inquiry which promises rich results to the investigator.

\section{Cransactions of 遇ramches.}

\section{SHROPSHIRE SCIENTIFIC BRANCH.}

A CASE OF LEUCOCYTHEMI A.*

By Winliam Eddowes, Esq., House-Surgeon to the Salop Infirmary.

[Read November 3rd, 1865.]

Samuer Williams, aged 28, a forgeman, single, a native of this county, was admitted into the Salop Infirmary, under the care of Dr. Beddoes, on September 16th, 1865.

The patient had enjoyed good health until last March (six months before admission), when he began to feel pain in the left hypochondriac region, not constant, but of a shooting character. Soon after this, he perceived some enlargement at the seat of pain. He continued to work for three weeks after the commencement of the symptoms, when he was obliged to give it up, and since has attempted to resume work without success, because exertion made him giddy.

Before admission, he appeared to have been treated for diabetes; and, upon that supposition, had been been put on a non-saccharine diet. Under this treatment, he continued to grow weaker and paler until admission.

Sept. 16th. The patient was of a light complexion, and remarkably pallid; the conjunctivæ were quite white; and the skin of the face and body looked as if totally deprived of all colouring matter. There was, however, no great emaciation. He complained of languor and drowsiness, as well as of great weakness and giddiness on exertion. There was occasional pain over the region of the spleen, which

* For the notes of this case, I am indebted to Mr. E. M. Little. could be felt distinctly enlarged both vertically and laterally. The liver and the rest of the viscer. seemed to be normal in size, and healthy. Tho inguinal and cervical glands were much enlarged. A little blood taken from the finger appeared much paler than usual; and the microscope showed that it contained almost as many colourless granular corpuscles as red. The urine contained neither sugar nor albumen; specific gravity 1011. He was ordered to have five grains of bromide of potassium three times a day.

Oct. 2nd. He was ordered a drachm of syrup of iodide of iron twice a day.

Oct. 9th. There was no improvement. He was dircoted to take fifteen minims of tincture of perchloride of iron and three minims of tincture of digitalis, in an ounce of water, three times a day.

Oct. 19th. Up to this date, notwithstanding the treatment, the patient continued to grow weaker. The spleen had not perceptibly altered in size.

Oet. 20th. He said that on the previous night he felt very cold, and was purged a good deal. Pulse 120 ; tongue dry, but clean. $\mathrm{He}$ was ordered to take a mixture of sulphate of quinine, sulphate of iron, and sulphuric ether; and a pint of porter.

Oct. 21st. Palse 124; tongue dry and clean. He stated that he felt intolerably hot during the night. The purging continued, and he had vomited up some dark matter like coffee-grounds. The skin was hot, but not very dry. He was ordered to have six ounces of whiskey.

R Tincturæ opii mxx; tincturæ catechu 3i; dococti hæmatox. $\zeta$ i. M. To be taken every four hours.

Oct. 22nd. Pulse 120. The tongue was dry and black, probably from the iron he was taking. Ho was purged less last night, but vomited several times, and had slight epistaxis. He had no appetite, and what he took was soon rejected. He complained much of dryness of the mouth and thirst. The temperature of the axilla was $102.3^{\circ}$.

\section{He died at 9 P.M.}

Sectio Cadaveris, twenty-four hours after death. The body was decidedly, but not extremely, emaciated. The inguinal and cervical glands were much enlarged. The lungs and heart were healthy. There were old adhesions of the right pleura. Both ventricles contained small yellowish coagula; and the veins generally were filled with coagula, looking like ordinary pus mixed with dark coloured blood. The liver was healthy and pale, and weighed 44 lbs. The gall-bladder was normal in size, and distended with dark coloured bile. The spleen was firm, paler than natural, weighing $1 \mathrm{lb} .6 \mathrm{oz}$; and old adhesions (so firm as to require cutting with a knife) bound it to the surrounding parts, especially the abdominal wall and left lobe of the liver. About the centre of the external surface was a yellowish-white-looking patch, of about the size of a half-crown, and extending into its structure about half an inch. It appeared to be a patch of organised lymph. The kidneys were healthy, but pale; and in the calyx of the right there was a considerable ecchymosis. The mesenteric glands, as well as those in the iliac fosse, were much enlarged from simple hypertrophy.

This well marked case of leucocythæmia presented very nearly the same history, course, termination, and morbid appearances, as many of those described by Dr. Hughes Bennett in his work on Medicine.

Professor Beale, to whom I sent some of the blood and spleen, kindly examined them, and reported that he considered them very beautiful specimens of the disease. 